\title{
SENSIBILIDADE DE CONJUNTOS DE NUVENS A VARIAÇÕES DE PARÂMETROS MICROFÍSICOS. PARTE I - SIMULAÇÃO DE CONTROLE
}

\author{
ANDRÉ DE SENA PINHEIRO, ALEXANDRE ARAÚJO COSTA \\ Universidade Estadual do Ceará (UECE), Fortaleza, CE, Brasil \\ andresenap@gmail.com, alexandrearaujoc@gmail.com
}

Recebido Julho de 2011 - Aceito Agosto de 2012

\begin{abstract}
RESUMO
Este trabalho é o primeiro de uma série de quatro artigos, com o objetivo de analisar como variações em parâmetros microfísicos afetam o ciclo de vida de nuvens de fase mista, para diferentes condições de estabilidade vertical, adotando como ferramenta uma versão de "cloud ensemble model" - CEM do Regional Atmospheric Modeling System (RAMS). São utilizados dados do Período de Obervação Intensiva (Intensive Observing Period - IOP) do Tropical Ocean Global / Atmosphere Coupled Ocean-Atmosphere Response Experiment (TOGA COARE) como forçante para o CEM. Este foi calculado para três regimes, a depender da atividade convectiva: "médio", em que foi considerada a média das variáveis para todo o IOP, "ativo" (convecção mais intensa) e "suprimido" (convecção menos pronunciada). Para cada regime, calculou-se a média das componentes zonal e meridional da velocidade do vento e as forçantes advectivas (horizontal mais vertical) de temperatura potencial e umidade específica. Tais forçantes foram assimiladas pelo CEM em diferentes testes de sensibilidade, descritos nas partes seguintes desta série de artigos. Na parte I, são apresentados os resultados referentes à simulação controle, mostrando como um CEM estabelece uma condição de quase-equilíbrio resultante da resposta da convecção às forçantes de grande escala estacionárias.
\end{abstract}

Palavras-chave: Convecção tropical, TOGA-COARE, Modelo de ensemble de nuvens, microfísica de nuvens.

\begin{abstract}
SENSITIVITY OF CLOUD ENSEMBLES TO VARIATIONS IN MICROPHYSICAL PARAMETERS. PART I - CONTROL SIMULATION

This work is the first from a series of four articles with the aim in analyzing how variations in microphysical parameters affect the life cycle of mixed-phase clouds, under different vertical stability conditions, using a Cloud Ensemble Model (CEM) version of the Regional Atmospheric Modeling System (RAMS) as a tool. Obtained data during the Intensive Observing Period (IOP) of the Tropical Ocean / Atmosphere Coupled Ocean-Atmosphere Response Experiment (TOGA COARE) were used as external forcing for the CEM. In order to represent different tropical environments, such forcing was calculated for three regimes, depending on the convective activity: "mean", in which the average variables over the entire IOP was used as forcing; "active", during which convection was stronger; and "suppressed", when convective activity was less pronounced. For each regime, the average of the zonal and meridional wind velocity, and advective forcing (horizontal plus vertical) of the potential temperature and specific humidity were calculated. Those forcings schemes were assimilated by the CEM in different sensitivity tests, described along the following parts of this series of articles. In Part I, results from a control simulation are presented, showing how the CEM establishes a quasiequilibrium state, resulting from the convective response to the stationary large scale forcing.
\end{abstract}

Keywords: Tropical convection, TOGA-COARE, Cloud ensemble model, Cloud microphysics 


\section{INTRODUÇÃO}

A existência de nuvens cobrindo parcela significativa da Terra é uma característica essencial de sua circulação geral atmosférica. A troposfera terrestre mantém um balanço de energia em grande parte, graças à liberação de calor latente associada à formação de nuvens e precipitação em contraposição ao resfriamento radiativo. Não obstante, modelos globais utilizados em simulações climáticas não conseguem representar explicitamente a formação de nuvens, sendo necessário parametrizar os processos que as envolvem, incluindo o transporte, aquecimento e secagem convectivos, a interação destas com os fluxos radiativos de onda curta e onda longa e com a camada-limite e, evidentemente, a produção de precipitação.

Tais parametrizações, entretanto, costumam não ter uma base física comum, sendo as proposições de fechamento, não raro, baseadas em considerações e/ou bases de dados diversas, o que constitui uma severa limitação dos modelos atmosféricos de grande escala.

Uma forma de minimizar esse problema é o uso de um modelo com maior resolução, sendo capaz de representar os fenômenos explicitamente e com os resultados aperfeiçoar tais parametrizações. Este é o centro da idéia defendida por Randall et al. (1996), Moncrieff et al. (1997) e outros autores, que propõem o uso de modelos de "conjunto" de nuvens (cloud ensemble models - CEM) como meio para construir parametrizações de processos de nuvens com base física comum.

Ainda segundo Randall et al. (1996), um CEM é um modelo com resolução alta o suficiente para resolver nuvens de forma individual (sem parametrização de convecção), com um domínio suficientemente grande para abrigar várias nuvens ao mesmo tempo e com um tempo de simulação longo o bastante para conter vários ciclos de vida de nuvens. Por vezes, este tipo de modelo foi usado na forma bidimensional (Costa et al., 2001a; Costa, 2004; Gao et al., 2006) apesar de, especialmente com os avanços na capacidade de processamento e armazenamento dos computadores atuais, funcionarem em 3D também. Eles calculam explicitamente variáveis difíceis de observar, tais como, a distribuição vertical de água líquida e gelo, fluxos de massa, fração de cobertura de nuvens (Randall et al., 1996).Além disso, ao representar processos microfísicos (nucleação, crescimento por difusão e colisão) e resolver circulações em escala de nuvens, são capazes de simular a interação do transporte convectivo com fluxos de superfície, turbulência e processos de transferência radiativa (Tao, 2007).

Muitos estudos que usaram este tipo de modelo foram direcionados à investigação das interações nuvem-radiação (Li et al., 1999), simulações de estado de quase-equilíbrio (Wu, 2002) ou investigação de processos de interação ar-mar em pequena escala, associado à presença de nuvens (Costa et al., 2001a,b; Costa 2004). Neste caso, em processos associados à convecção atmosférica, o estado do oceano pode ser modificado, sendo necessário, portanto um acoplamento com um modelo oceânico, como sugerido em Moncrieff et al. (1997). É o que fez Costa et al. (2001b), para evidenciar que uma variabilidade oceano-superfície pode ser gerada por nuvens, que por sua vez, acarretam anomalias de água doce via precipitação e indução não-homogênea do estresse do vento e forçante radiativa. Já Gao et al. (2006) usaram um modelo 2D acoplado com um modelo oceânico, para analisar as variações diurnas e processos dominantes de microfísica responsáveis pelo desenvolvimento de nuvens quentes e frias e de precipitação em um regime convectivo profundo.

Outra aplicação dos CEM's é a substituição da parametrização de convecção em modelos globais, constituindo a chamada superparametrização ou Cloud Resolving Convective Parameterization, CRCP, (Grabowski e smolarkiewicz, 1999; Grabowski, 2003, 2004, Khairoutdinov et al. 2005; Madja, 2007; Zhu et al., 2009). Segundo Grabowski e Smolarkiewicz (1999), a estratégia do CRCP é considerar dois modelos atmosféricos distintos acoplados entre si de uma forma particular. O primeiro é um modelo do escoamento de grande escala tridimensional, tipicamente um modelo de circulação geral (restrito à dinâmica), com espaçamento de grade horizontal de aproximadamente 100 $\mathrm{km}$. O segundo modelo é um modelo bidimensional que resolve a escala de nuvens, embutido em cada coluna do modelo de grande escala. O modelo de escala de nuvens geralmente usa a mesma grade vertical do modelo de grande escala, mas com uma resolução horizontal mais alta (espaçamento da grade em torno de $1 \mathrm{~km}$ ), podendo assim, de acordo com Grabowski (2004) resolver explicitamente os fenômenos de pequena escala em cada coluna do modelo de escala global tendo, desse modo, uma representação mais realista da convecção. Este tipo de modelo é usado, portanto, em certa medida, como uma parametrização convencional, no sentido de que ele recebe as tendências devido a processos de grande escala (ou de escala resolvida) como entrada, e, como saída, retorna as tendências devidas a processos na escala de sub-grade, obtidas pela média horizontal de campos do CEM. A principal diferença em relação às parametrizações convencionais é que, diferentemente desta, o CEM pode explicitamente resolver a maior parte da dinâmica de nuvens e interações destas com processos físicos até uma escala definida pela resolução do próprio modelo e, assim sendo, espera-se que ele responda de uma forma mais realista à forçante de grande escala aplicada (Khairoutdinov et al., 2005).

Esta série de artigos tem como objetivo estudar a influência dos aerossóis em nuvens convectivas tropicais e, mais especificamente, analisar os possíveis impactos na formação e no ciclo de vida destas nuvens, bem como o efeito sobre a precipitação formada, para diferentes condições de estabilidade 
vertical. Na Parte I, apresentamos uma descrição do modelo numérico, assim como dos dados utilizados para forçá-lo, das condições de inicialização e a configuração do mesmo para a simulação de controle (Seção 2). Os resultados desta simulação mostram como um CEM estabelece uma condição de quaseequilíbrio ao ser forçado por tendências advectivas de grande escala (Seção 3), o que é crucial para verificar a habilidade do modelo em representar a interação da convecção com a grande escala. Ao final, são discutidas as implicações e apontadas incertezas a serem exploradas nas partes subsequentes (Seção 4).

\section{METODOLOGIA}

\subsection{Dados observados do TOGA-COARE}

O Tropical Ocean Global Atmosphere (TOGA) Coupled Ocean-Atmosphere Research Experiment (COARE) foi um programa internacional de pesquisa que investigou fenômenos associados à interação entre a atmosfera e o oceano na região do Pacífico Ocidental, partindo da prerrogativa que as perturbações provenientes desta região influenciam processos como o El Niño-Oscilação Sul. A região do experimento, com os sítios observacionais, é mostrada na Figura 1, com destaque para a área interna, a matriz de fluxos intensivos (Intensive Flux Array - IFA), em que se concentraram os esforços do período de observação intensiva (Intensive Observing Period, IOP), entre novembro de 1992 e fevereiro de 1993. O IOP envolveu a utilização de vários instrumentos de monitoramento meteorológico e oceanográfico desde bóias e navios, até radares Doppler terrestres e aeronaves equipadas com radar Doppler. O experimento compreendeu estudos envolvendo a estrutura da

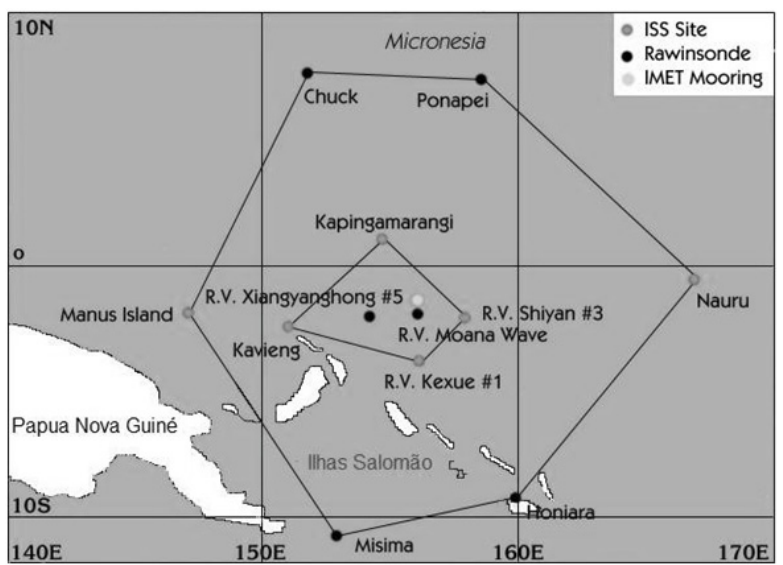

Figura 1 - Matrizes observacionais do experimento TOGA COARE: Matriz de larga escala (large-scale array - LSA) $(140 \mathrm{~L}-180$ e $10 \mathrm{~N}$ - 10S), matriz de sondagem externa (outer sounding array - OSA) (polígono intermediário) e matriz de fluxo intensivo (intensive flux array - IFA) (polígono mais interno à figura). atmosfera (Mcbride et al., 1995) e oceano (You, 1995) e sobre a organização da convecção e precipitação (Short et al., 1997).

Neste trabalho, serão utilizados os dados de sondagem sobre a IFA, coletados durante o IOP, estando as variáveis em níveis de pressão para encontrar as forçantes de grande escala (velocidade zonal e meridional e advecção de temperatura e umidade) que alimentaram o CEM. Parte das simulações nesta sequência de artigos utilizou uma forçante que corresponde à média dessas variáveis durante todo o IOP, que foi chamado de período médio. Para outras, utilizando como critério a temperatura de brilho, foram separados os períodos de maior atividade convectiva, classificado como período ativo e os de convecção suprimida (menor atividade convectiva), isto é, foi identificada a terça parte do período (não contínuo) no qual as temperaturas de brilho possuem valores mais baixos, significando um topo de nuvem mais alto (convecção ativa) e a terça parte com valores maiores, indicando nuvens com topo mais baixo (convecção suprimida).

\subsection{Modelo de "Ensemble" de nuvens}

O modelo CEM utilizado neste trabalho terá configurações baseadas em Costa et al. (2001a) e Costa (2004). trata-se de uma versão modificada do Regional Atmospheric Modeling System-RAMS (Pielke et al., 1992; Cotton et al., 2003), sendo usada apenas uma grade, localizada no oceano sobre a linha do equador. a grade é bidimensional, com projeção cartesiana e fronteiras laterais cíclicas; tem 1024 pontos na direção x e 70 pontos de grade na direção z com espaçamentos de grade de $1000 \mathrm{~m}$ para $\mathrm{x}$ e variável para $\mathrm{z}$, partindo de $80 \mathrm{~m}$ para o primeiro nível (partindo da superfície) com razão de aumento vertical de 1,036 , sendo limitado a um espaçamento de $600 \mathrm{~m}$. a variação da radiação ao longo da longitude não é considerada, sendo parametrizada segundo Chen-Cotton, e chamada a cada 600 segundos. a parametrização de turbulência segue o esquema de Smagorinsky (1963). o nudging é desligado, sendo introduzida uma subrotina no código do modelo que calcula um nudging não local baseado nas equações descritas em Grabowski et al. (1996) para o momentum:

$$
\begin{aligned}
& \left.\frac{\partial u}{\partial t}\right|_{F G E}=-\frac{\bar{u}-u_{0}}{\tau_{m}} \\
& \left.\frac{\partial v}{\partial t}\right|_{F G E}=-\frac{\bar{v}-v_{0}}{\tau_{m}}
\end{aligned}
$$

Esse tipo de nudging, ao mesmo tempo em que mantém a média do vento no domínio do CEM muito próxima das observações, permite o desenvolvimento livre de padrões de menor escala, visto que ele atua sobre os campos médios 


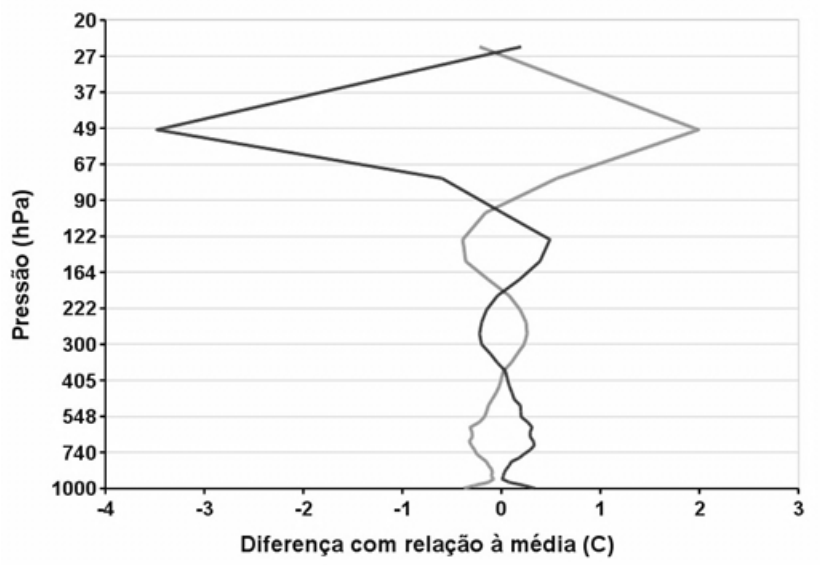

Figura 2 - Perfis da diferença de temperatura entre perfil ativo e suprimido. Em cinza escuro a diferença entre perfil ativo e médio e em cinza claro a diferença entre perfil suprimido e médio.

horizontais, e não sobre cada ponto de grade do modelo (Costa, 2004).

Em todas as simulações nesta série de artigos, o modelo é inicializado homogêneo, a partir da média das sondagens sobre a IFA durante o IOP, de novembro de 1992 a fevereiro de 1993. A temperatura de superfície do mar foi mantida constante e igual a 302,54K, correspondendo à média sobre o IFA durante o IOP não sendo atualizada ao longo da simulação. Junto com o nudging, é acrescentada uma perturbação aleatória no código do modelo (como este é iniciado homogêneo, faz-se necessário um fator para que as nuvens possam desenvolver-se durante a simulação) durante os primeiros 60 s de simulação.

\subsection{Simulação de controle}

Na simulação de controle, a evolução da temperatura potencial e da umidade específica seguem as forçantes mostradas nas Figuras 4a e 5a, isto é, as médias para todo o IOP. O nudging não-local do vento (Equações 1 e 2) é feito utilizando as componentes mostradas no painel 3a. Valores padrão do RAMS foram utilizados nas opções da microfísica de nuvens, o que implica no uso do esquema de um momento (Walko et al., 1995), com concentração de gotículas especificada de 300 $\mathrm{cm}^{-3}$, diâmetros especificados de $1 \mathrm{~mm}$ para gotas de chuva, neve, agregados e graupel e $3 \mathrm{~mm}$ para granizo e parâmetro de forma igual a 2 para todas as espécies de hidrometeoros. Apenas para a categoria de gelo primário utilizou-se um esquema de dois momentos (Meyers et al., 1997).

Assim como nas demais, na simulação de controle, o CEM representou 15 dias de interação dos sistemas de nuvens com as forçantes de grande escala, sendo que os 3 primeiros dias de transiente são descartados e apenas os 12 dias seguintes são utilizados para gerar as estatísticas analisadas nesta série de artigos, em que o primeiro dia de simulação coincide com o primeiro dia do IOP.

\section{RESULTADOS}

\subsection{Forçantes de grande escala}

Os forçantes de grande escala são calculados a partir dos dados da matriz de fluxo intensivo (IFA) e alimentarão o CEM durante a simulação.

As diferenças entre os perfis de temperatura para os casos ativo e suprimido e a média são mostradas na Figura 2. Essa figura evidencia a diferença de comportamento na estabilidade vertical dos dois casos, sendo a convecção ativa, o período de maior instabilidade durante o experimento (temperaturas maiores que a média desde a superfície até o nível próximo de $400 \mathrm{hPa}$ ), e o de convecção suprimida, de pouca instabilidade (temperaturas menores que a média até o nível próximo de $400 \mathrm{hPa}$ ).

As médias das componentes zonal e meridional da velocidade para todo o período e para as fases ativa e suprimida são mostradas na Figura 3. Estas médias serão usadas para o cálculo do nudging não-local nas simulações. Percebe-se que o escoamento, em qualquer dos casos, é praticamente zonal e, diferentemente do que ocorre na maioria das regiões tropicais, o vento em baixos níveis é de oeste, o que é mais acentuado no período ativo (Figura $3 b$ ) devido ao fato de esse período registrar maior convergência de grande escala.

A grande escala atua na atmosfera resfriando e umedecendo o ambiente. Em virtude desse resfriamento, a convecção reage com a formação de nuvens que aquecem o ambiente, via liberação de calor latente, ao mesmo tempo em que o secam, consumindo o vapor disponível durante a condensação e removendo água da atmosfera ao produzir precipitação. Sobre o Pacífico Oeste, esse sinal é percebido no perfil médio de advecção de temperatura potencial (Figura 4a), em que o termo vertical é positivo em praticamente toda a troposfera, indicando movimento ascendente e resfriamento de grande escala. No regime ativo, para a advecção de temperatura potencial (Figura $4 \mathrm{~b}$ ), percebe-se um resfriamento de grande escala ainda maior, forçando uma atividade convectiva mais intensa, favorecendo o aparecimento de nuvens de desenvolvimento vertical acentuado, responsáveis por forte precipitação. Por outro lado, no regime suprimido (Figura 4c), até aproximadamente $500 \mathrm{hPa}$, o resfriamento é praticamente nulo, chegando a ocorrer um aquecimento de grande escala desse nível até cerca de $160 \mathrm{hPa}$. O resultado é a subsidência de grande escala, inibindo a formação de nuvens precipitantes e, em geral, permitindo apenas convecção rasa.

A Figura 5 mostra a média para a advecção de umidade no conjunto do experimento (Figura 5a) e nos casos ativo (Figura $5 b$ ) e suprimido (Figura 5c). O Pacífico oeste, em média, é 
mais úmido do que suas vizinhanças e consequentemente, o ar transportado para essa região, via advecção horizontal é menos úmido e este termo produz secagem em todos os casos. Já o transporte vertical do ar nessa região, carrega o ar úmido para cima, alimentando a atmosfera com o vapor disponível próximo à superfície naquela região. $\mathrm{O}$ resultado dessa advecção de umidade horizontal e vertical é um umedecimento médio do ambiente (Figura 5a), que se acentua no período ativo (Figura $5 b$ ). No período suprimido, o umedecimento fica restrito a porção inferior da atmosfera.

\subsection{Sensibilidade do CEM}

Para analisar os resultados de simulações de sensibilidade do CEM, é necessário que o modelo produza estatísticas estacionárias, ou seja, esteja em estado de quase equilíbrio. As Figuras 6 e 7, respectivamente, apresentam a evolução temporal da razão de mistura de vapor d'água e temperatura potencial equivalente $\left(\theta_{\mathrm{e}}\right)$ em determinados níveis do modelo para os 12 dias finais de simulação. Na Figura 6, verifica-se que a razão de mistura de vapor d'água apresenta oscilações de pequena amplitude em torno de valores ligeiramente abaixo de $18 \mathrm{~g} / \mathrm{kg}$

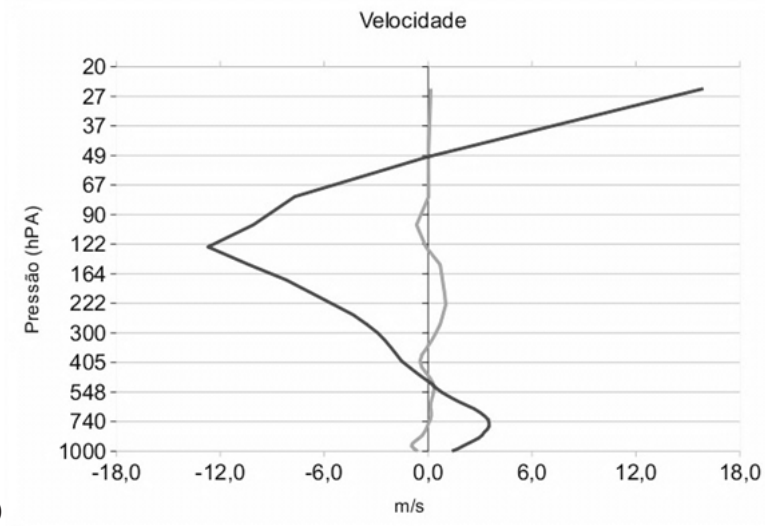

a 70m, em torno de $12 \mathrm{~g} / \mathrm{kg}$ a $1246 \mathrm{~m}$ e ligeiramente abaixo de 5 $\mathrm{g} / \mathrm{kg}$ a $4527 \mathrm{~m}$ acima da superfície. Na Figura 7, são apresentadas as séries de $\theta_{\mathrm{e}}$ nos níveis de $1246 \mathrm{~m}$ (baixa troposfera), $4527 \mathrm{~m}$ (média troposfera) e $10288 \mathrm{~m}$ (alta troposfera), com esta variável oscilando aproximadamente em torno de 339K, 347K e $348 \mathrm{~K}$, respectivamente. Como era de se esperar, a temperatura potencial equivalente apresenta valores mais elevados na baixa e na alta troposfera, e valores menores na troposfera média. Novamente, o modelo não exibe tendência nessa variável, que tende a ficar estável nesse período de 12 dias como resultado da resposta convectiva à forçante de grande escala.

São mostradas também as séries temporais para a taxa de precipitação (Figura 8), fluxos de radiação (Figura 9) e fluxo de calor (Figura 10), além de figuras do condensado médio instantâneo como função de x e z, para evidenciar a formação de nuvens em dois instantes: $\mathrm{t}=168 \mathrm{~h} \mathrm{e} \mathrm{t}=192 \mathrm{~h}$ (Figura11).

A série temporal para a taxa de precipitação mostra-se coerente com a existência de um ciclo diurno da convecção, mesmo sendo o forçante de grande escala e a temperatura da superfície mantidos constante. Isto sugere que a interação entre nuvens e radiação é fundamental no controle do desenvolvimento da convecção.

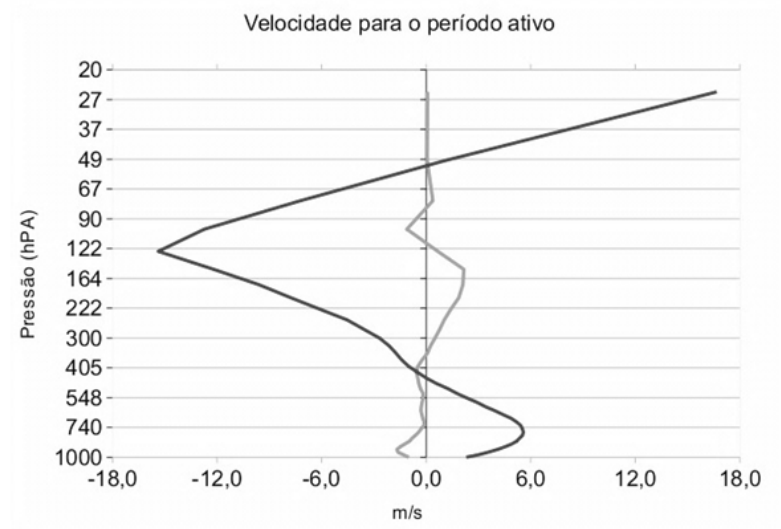

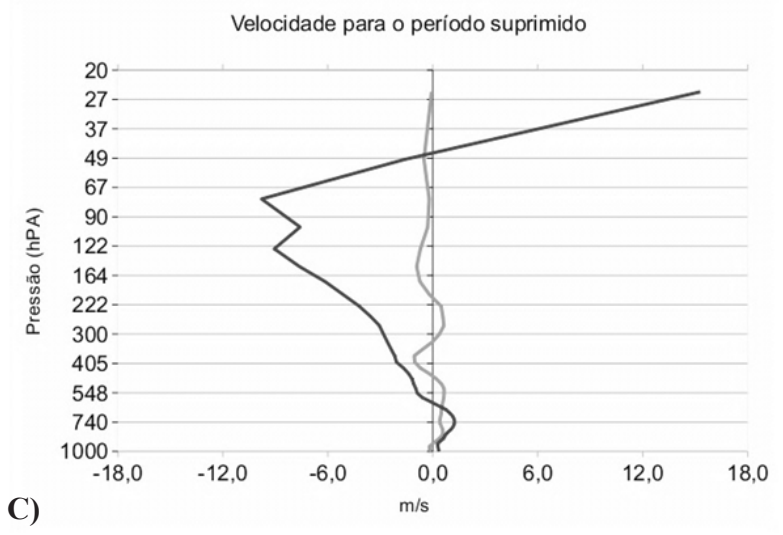

Figura 3 - Médias das componentes zonal e meridional da velocidade do vento para (a) todo o período, (b) fase ativa e (c) fase suprimida. Na figura, a componente zonal está em cinza escuro e a meridional em cinza claro. 
Há diversos eventos em que tanto a radiação de onda curta (Figura 9a), quanto a radiação de onda longa na superfície (Figura 9b), apresentam-se coerentes com a precipitação. Evidentemente, durante o dia, uma maior presença de nuvens no domínio do modelo (e presumivelmente uma maior taxa média de precipitação) implica na redução da radiação solar incidente sobre a superfície. No caso da radiação de onda longa, uma maior quantidade de nuvens tem como consequência mais emissão de infravermelho para baixo.

Para a radiação de onda curta, uma maior quantidade de nuvens é acompanhada de um maior bloqueio da radiação solar. Sobre o continente, ao se reduzir a radiação incidente, se diminuiria o aquecimento da superfície, diminuindo a instabilidade da atmosfera, inibindo a convecção e fazendo com que as nuvens fossem dissipadas. No entanto, no nosso caso, em que idealizamos condições oceânicas com TSM constante, isso não ocorre. Como exemplo, percebe-se, na Figura 9a, que, ao compararmos o instante $\mathrm{t}=168 \mathrm{~h}$ (maior atividade convectiva, como mostrado na Figura 11a) com o instante $t=192 \mathrm{~h}$ (menor atividade convectiva) da simulação controle, a máxima radiação de onda curta aumenta durante esta última, para uma mesma hora do dia.

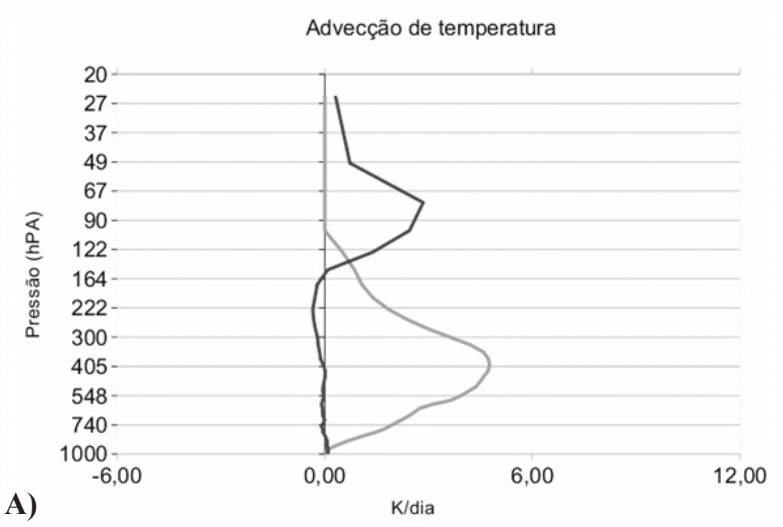

No que diz respeito à radiação de onda longa, tomando como referência, por exemplo, as horas 168 e 192 da simulação controle, vê-se que um maior fluxo de radiação ocorre simultaneamente a uma maior taxa de precipitação (pouco antes da hora 168), reduzindo-se à medida que avançamos na simulação até chegar na hora 192. A partir daí, há um novo crescimento no fluxo da radiação de onda longa, acompanhando novo aumento na taxa de precipitação.

No entanto, a relação entre a existência de uma cobertura de nuvens maior (o que está relacionado com um aumento da radiação de onda longa que chega à superfície) e a ocorrência da precipitação não é tão simples. Daí, nem sempre se percebe uma relação direta entre os máximos de precipitação e o fluxo radiativo de onda longa. De fato, a correlação temporal entre essas duas variáveis chega apenas a 0,29 , com defasagem de uma hora entre elas (precipitação à frente).

Os fluxos de calor latente e sensível (Figura 10) seguem um comportamento esperado. Os valores de calor latente são praticamente uma ordem de magnitude maiores do que os de calor sensível. Além disso, como a região do TOGA-COARE é caracterizada por ventos de grande escala relativamente fracos, há uma relação intrínseca entre fluxos de superfície e

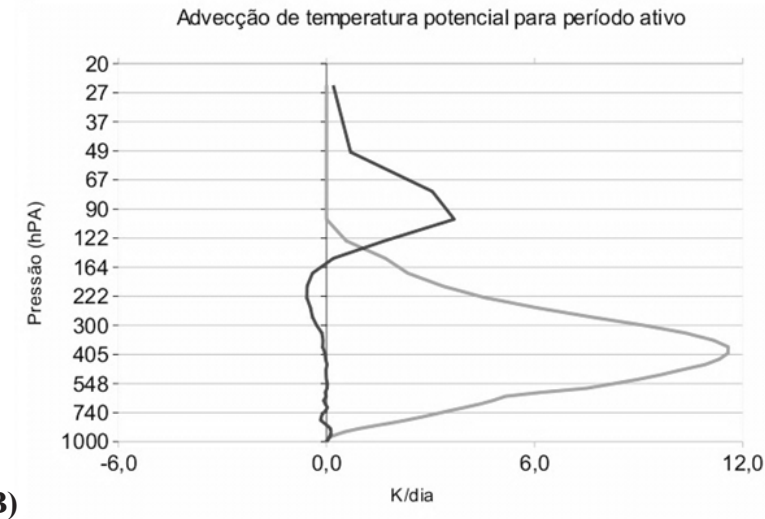

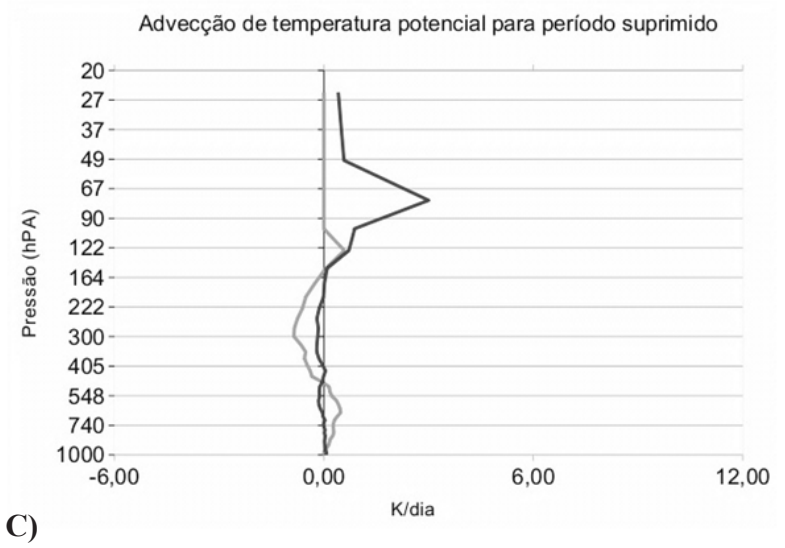

Figura 4 - Médias da advecção das temperaturas potenciais para (a) todo o período, (b) fase ativa e (c) fase suprimida. Na figura, a advecção horizontal está em cinza escuro e a vertical em cinza claro. 

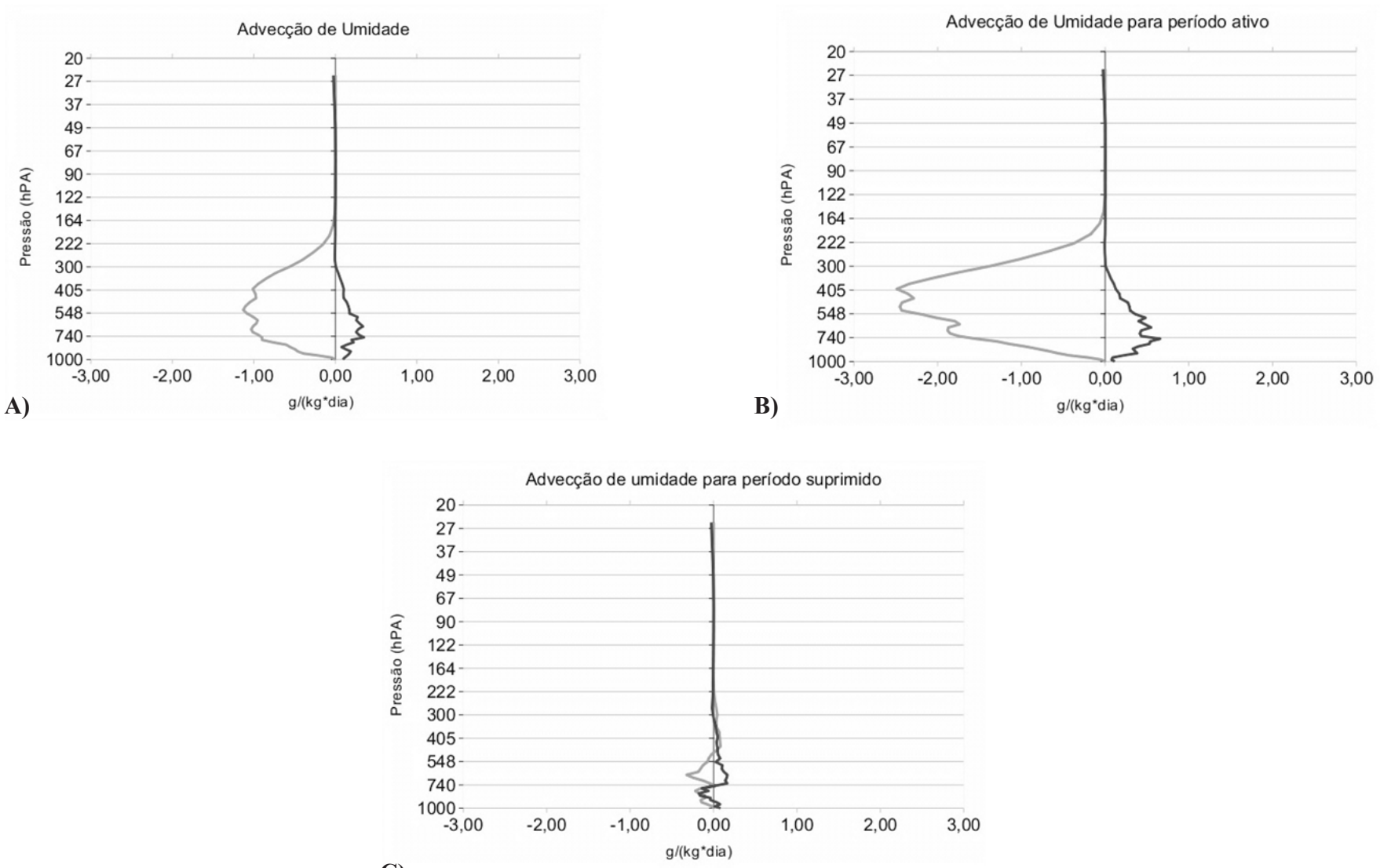

C)

Figura 5 - Perfis de advecção horizontal de umidade (em cinza escuro) e advecção vertical de umidade (em cinza claro).

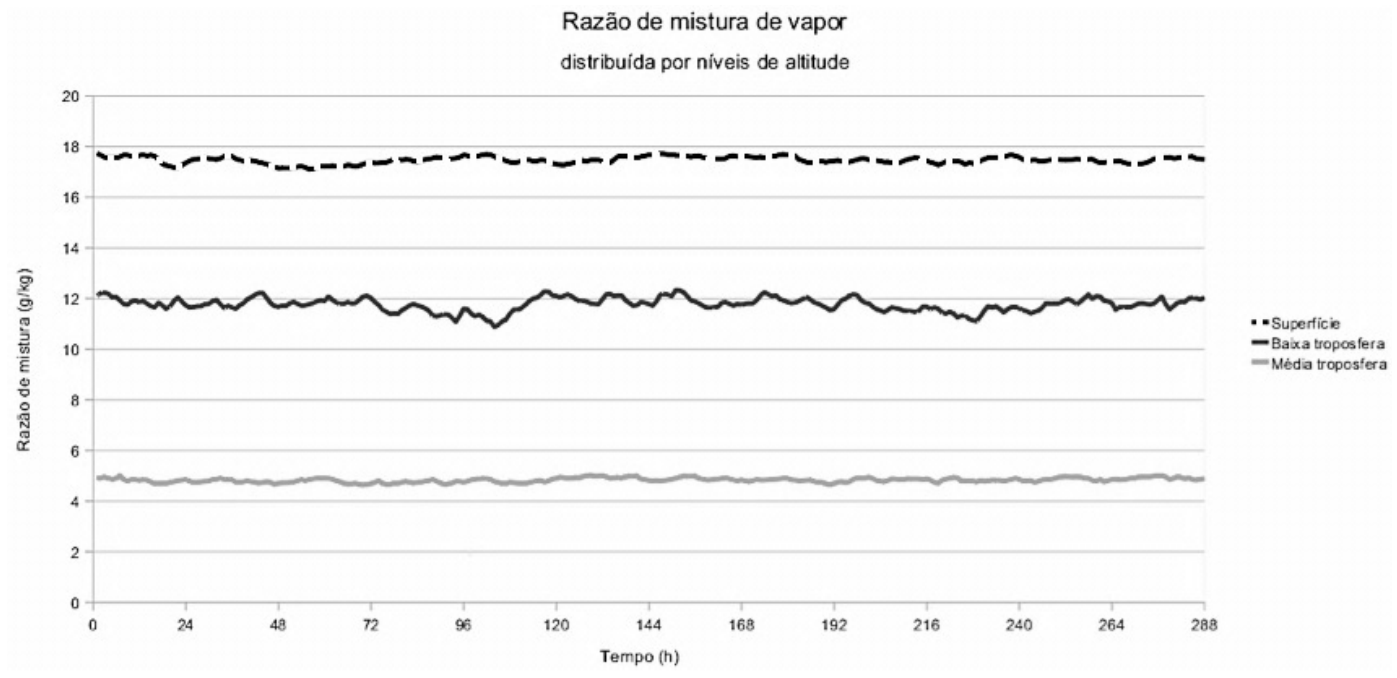

Figura 6 - Série temporal da razão de mistura de vapor em diferentes níveis de altitude do modelo: próximo à superfície (70 m), baixa troposfera (1246 m) e média troposfera $(4527 \mathrm{~m})$. 


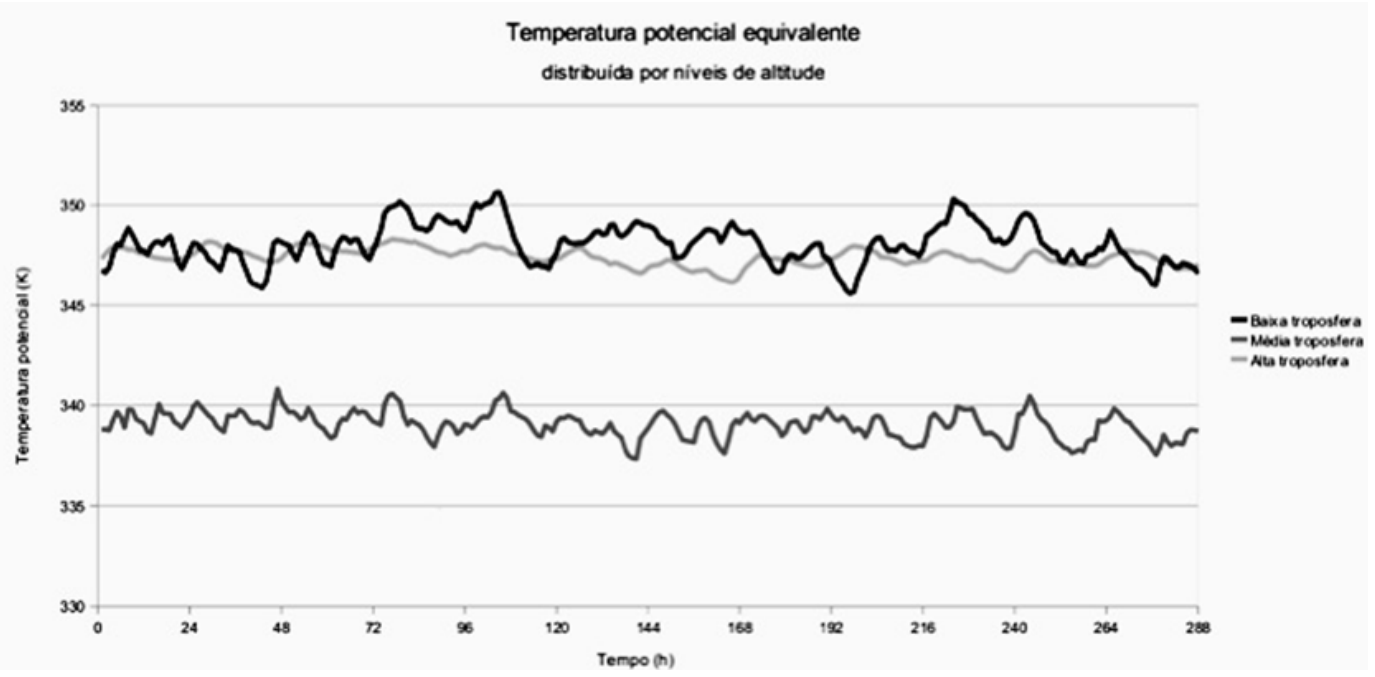

Figura 7 - Série temporal da temperatura potencial equivalente em diferentes níveis de altitude do modelo: baixa troposfera (1246 m), média troposfera $(4527 \mathrm{~m})$ e alta troposfera $(10288 \mathrm{~m})$.

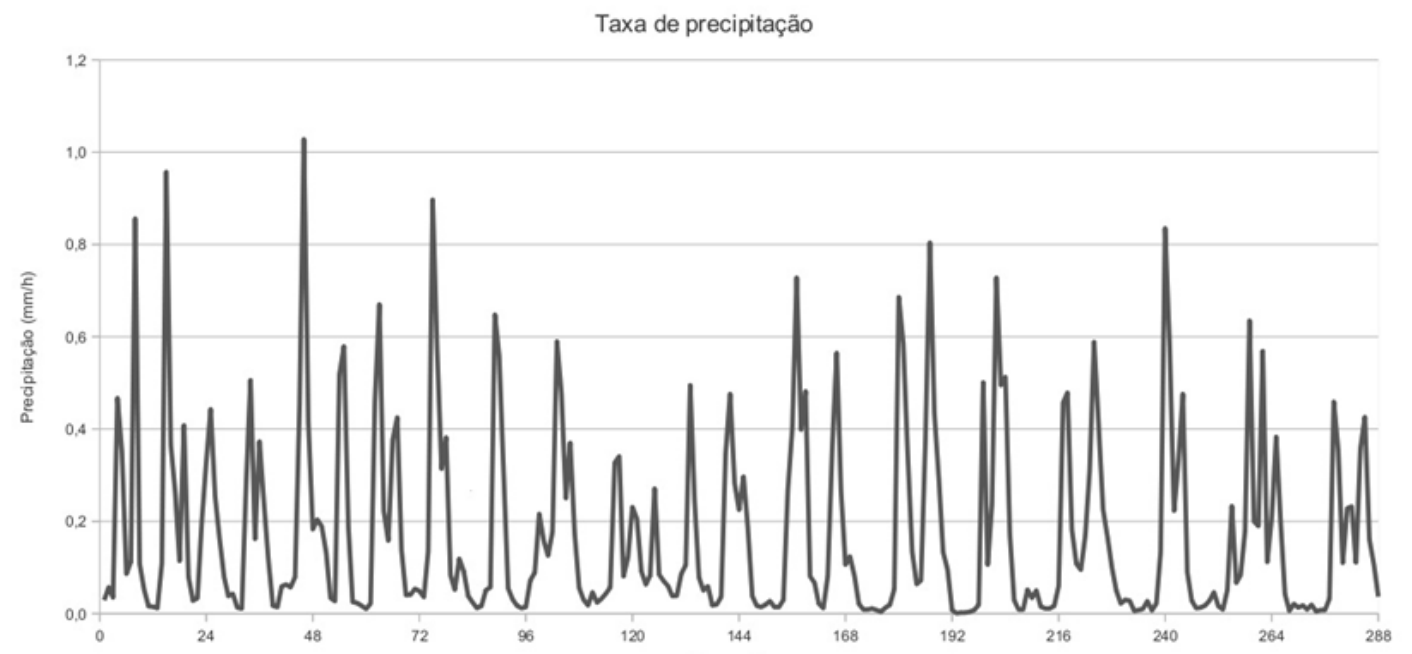

Figura 8 - Taxa de precipitação $(\mathrm{mm} / \mathrm{h})$ ao longo da simulação controle. Média sobre o domínio do modelo.

precipitação. Como sugerido por Costa et al. (2001b), isto ocorre porque a ocorrência de rajadas de vento de pequena escala está associada à precipitação, impactando os fluxos médios de calor. Quando da ocorrência de chuva mais intensa, o que propicia o aparecimento de correntes descendentes mais fortes, aumentando substancialmente o efeito de rajada sobre a superfície do oceano, cresce o fluxo de calor. As correlações entre precipitação e fluxos de calor na simulação controle são altas: 0,56 para o fluxo de calor latente (sem defasagem) e 0,58 para o fluxo de calor sensível (defasagem de uma hora, com a precipitação precedendo).

\section{CONSIDERAÇÕES FINAIS}

Modelos de ensemble de nuvens foram desenvolvidos principalmente a partir dos anos 90 , tendo sido seu uso incentivado por iniciativas como o GCSS (GEWEX Cloud System Study). Hoje são ferramentas bem estabelecidas junto à comunidade científica de modeladores atmosféricos. De um lado, este tipo de modelo é limitado por não interagir com a grande escala (já que esta é prescrita). No entanto, de outro, ao resolver explicitamente as nuvens, ele permite investigar processos como a interação entre as nuvens e a radiação, entre as nuvens e a camada-limite, etc. Assim, resultados de simulações utilizando modelos de ensemble de nuvens passaram a ser utilizados na construção de parametrizações fisicamente mais consistentes para modelos de escala maior, bem como, inseridos diretamente em modelos de circulação geral, em substituição aos esquemas de convecção tradicionais ("super-parametrização").

Neste primeiro artigo de uma série, apresentamos resultados com um modelo de ensemble de nuvens forçado por 

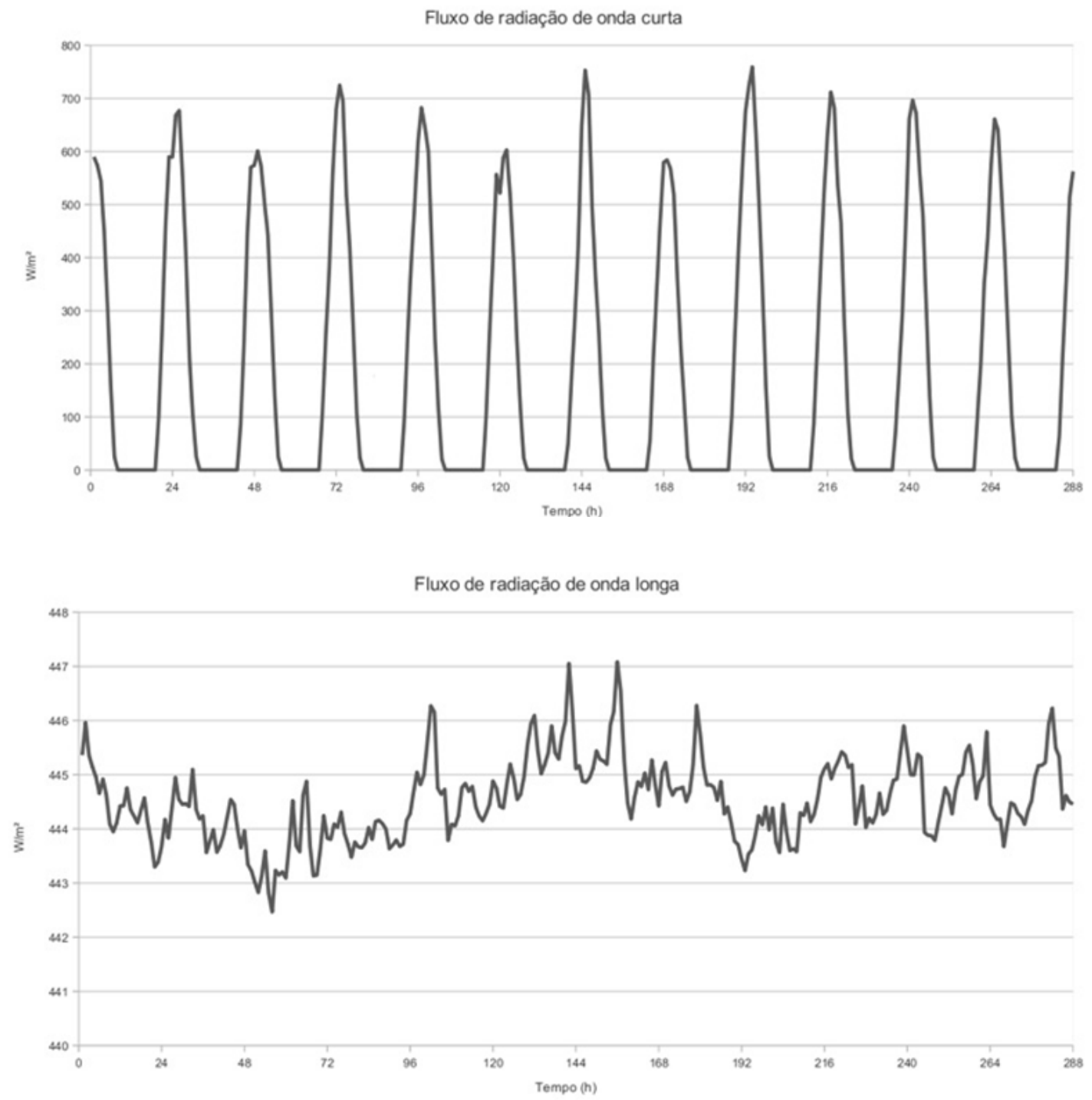

Figura 9 - Fluxos (em W.m-2) de (a) radiação de onda curta e (b) radiação de onda longa. Médias sobre o domínio do modelo.

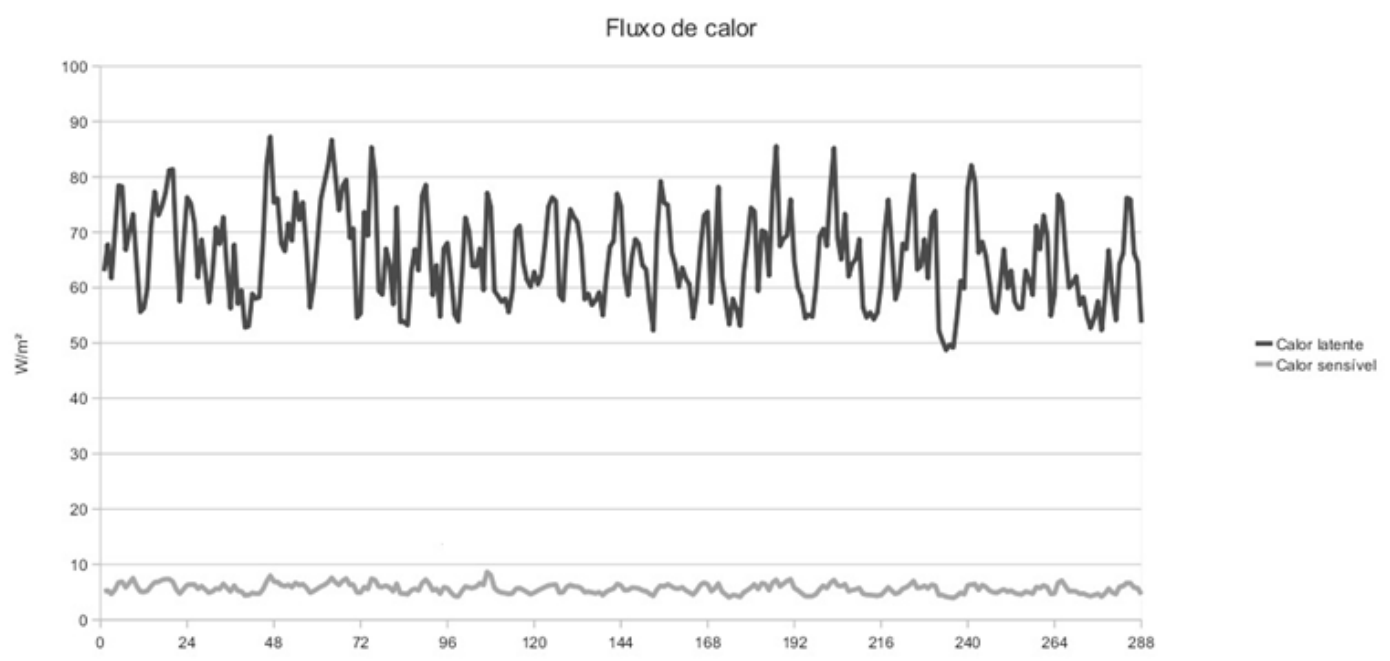

Figura 10 - Fluxos (em W.m-2) de calor latente e sensível. Médias sobre o domínio do modelo. 

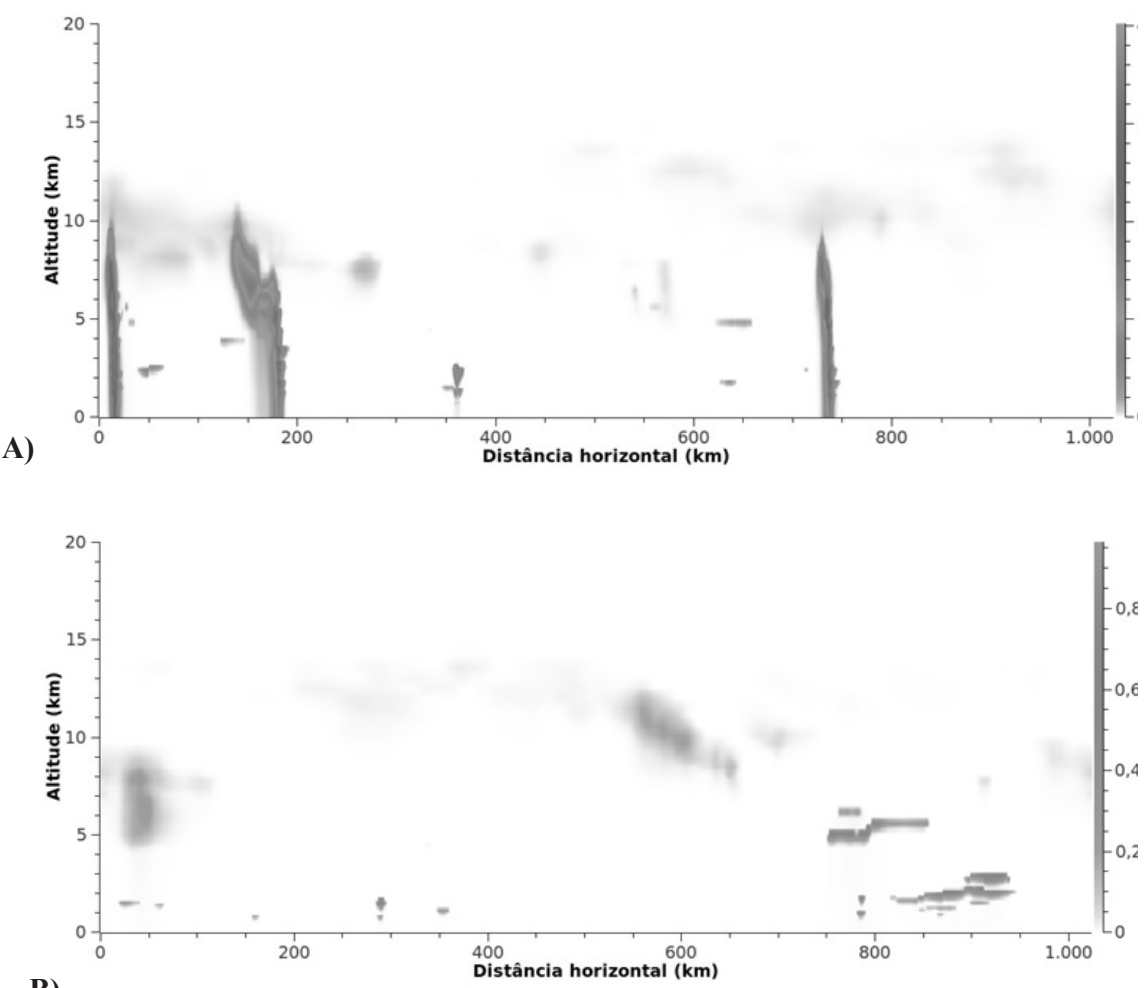

B)

Figura 11 - Razão de mistura do condensado total $(\mathrm{g} / \mathrm{kg})$ nos instantes (a) $\mathrm{t}=168 \mathrm{~h}$ e (b) $\mathrm{t}=192 \mathrm{~h}$, correspondendo a instantes de intensa e de reduzida atividade convectiva, respectivamente.

dados de grande escala correspondentes ao estado médio da atmosfera sobre o TOGA-COARE IFA durante o IOP. O objetivo principal foi verificar a habilidade do modelo em estabelecer um regime de quase-equilíbrio, com a convecção explicitamente resolvida se desenvolvendo em resposta ao umedecimento e resfriamento de grande escala e, via condensação seguida de precipitação, compensando esses processos, ao remover vapor d'água e liberar calor latente. De fato, logo nos primeiros dias de simulações efetuadas com uma forçante de grande escala estacionária, o modelo simulou a formação de nuvens que assegurou um estado de quase equilíbrio, não produzindo tendências de longo prazo nas médias sobre o domínio de variáveis atmosféricas médias, como razão de mistura de vapor e temperatura potencial equivalente. Interações nuvem-radiação foram particularmente importantes, com surgimento de um ciclo diurno da precipitação associado exclusivamente a estas, visto que tanto a forçante de grande escala, quanto a temperatura da superfície do mar foram mantidas constantes ao longo de todo o período de simulação.

Os resultados indicam, portanto, que o modelo pode ser utilizado para investigar interações nuvem-radiação. A representação dos processos de evolução do campo de nuvens e como este interage bidireccionalmente com a radiação é bastante inadequada nos modelos de circulação geral atmosférica que compõem os modelos do sistema terrestre utilizados em simulações climáticas. Isto contribui para que a incerteza na forçante radiativa associada às alterações antropogênicas sobre o campo de aerossóis seja bastante pronunciada.

Uma vez demonstrado, portanto, a utilidade desta ferramenta, os artigos seguintes desta série tratarão da sensibilidade do modelo de ensemble de nuvens a alterações nos parâmetros microfísicos (especificamente a concentração de gotículas, as dimensões dos hidrometeoros de fase sólida e mista e a forma da distribuição de hidrometeoros), além do ciclo diurno da convecção tropical abordado em vários artigos tais como Machado et al. (2004), Rickenbach (2004), Martins et al., (2010) entre outros. Nesses estudos seguintes, será dada ênfase ao impacto dessas alterações microfísicas sobre o balanço de radiação.

\section{AGRADECIMENTOS}

O presente trabalho foi financiado pelo CNPq, através do projeto CONBINE Convecção no Nordeste Brasileiro: Interações em Múltiplas Escalas), processo 620057/2008-4. $\mathrm{O}$ primeiro autor agradece à Fundação Cearense de Apoio ao Desenvolvimento Científico e Tecnológico - FUNCAP - pela concessão de bolsa de mestrado para desenvolvimento de seus 
trabalhos junto ao Mestrado Acadêmico em Ciências Físicas Aplicadas da Universidade Estadual do Ceará.

\section{REFERÊNCIAS}

COSTA, A. A. cloud-ensemble simulations of atmospheric convection over the ocean. Part I: model description, coupled and uncoupled simulations of a TOGA-COARE case. Revista Brasileira de Meteorologia, v. 19, n. 3, p.227-236, 2004.

COSTA, A. A.; COTTON, W. R.; WALKO, R. L.; PIELKESr., R. A. SST Sensitivities in Multiday TOGA COARE CloudResolving Simulations. Journal of the Atmospheric Sciences, v. 58, p. 253-268, 2001a.

COSTA, A. A.; COTTON, W. R.; WALKO, R. L.; PIELKE, R. A. Sr. Coupled Ocean-Cloud-Resolving Simulations of the Air-Sea Interaction over the Equatorial Western Pacific. Journal of the Atmospheric Sciences, v. 58, p. 3357-3375, $2001 b$.

COTTON, W. R.; PIELKE, R. A. Sr.; WALKO, R. L.; LISTON, G. E.; TREMBACK, C. J.; JIANG, H.; MCANELLY, R. L.; HARRINGTON, J. Y.; NICHOLLS M. E.; CARRIO, G. G.; MCFADDEN, J. P. RAMS 2001: Current status and future directions. Meteorology and Atmospheric Physics, v. 82, p. 5-29, 2003.

GAO, S.; PING, F.; LI, X. Cloud microphysical process associated with the diurnal variations of the Tropical convection: a 2D cloud resolving modeling study. Meteorogy and Atmospheric Physics, v. 91, p. 9-16, 2006.

GRABOWSKI, W. W. Impact of cloud microphysics on convective-radiativequase equilibrium revealed by cloudresolving convection parameterization. Journal of Climate, v. 16, p. 3463-3475, 2003.

GRABOWSKI, W. W. An improved framework for superparameterization. Journal of the Atmospheric Sciences, v. 61, p. 1940-1952, 2004.

GRABOWSKI, W. W., SMOLARKIEWICZ, P. K. CRCP: a cloud resolving convection prameterization for modeling the tropical convective atmosphere. Physica D. v. 133, p. 171-178, 1999.

GRABOWSKI, W. W.; WU, X.; MONCRIEFF M. W. Cloudresolving modeling of Tropical cloud systems during Phase III of GATE. Part I: two-dimensional experiments. Journal of the Atmospheric Sciences, v. 53, n. 24, p. 3684-3709, 1996.

KHAIROUTDINOV, M.; RANDALL, D.; DEMOTT, C. Simulations of the Atmospheric general circulation using a cloud-resolving model as superparameterization of physical process. Journal of the Atmospheric Sciences, v. 62, p.2136-2154, 2005.
LI, X.; SUI, C.-H.; LAU, K.-M.; CHOU, M.-D. Large-scale forcing and cloud-radiation interaction in the tropical deep convective regime. Journal of the Atmospheric Science, v. 56, p. 3028-3042, 1999.

MACHADO, L.A.T.; LAURENT, H.; DESSAY N.; MIRANDA, I. Seasonal and diurnal variability of the convection over the Amazonia: A comparison of different vegetation type and large scale forcing. Theoretical and Applied Climatology, v. 78, n. 1-3, p. 61-77. 2004.

MADJA, A. J. Multiscale models with moisture and systematic strategies for superparameterization. Journal of the Atmospheric Sciences, v. 64, p. 2726-2734, 2007.

MARTINS, R.C.G.; MACHADO, L.A.T.; COSTA, A.A. Characterization of the microphysics of precipitation over Amazon region using radar and disdrometer data. Atmospheric Research, v. 96, p 388-394. 2010.

MCBRIDE, J. A.; DAVIDSON, N. E.; PURI, K.; TYRREL, G. C.The flow during TOGA COARE as diagnosed by the BMRC Tropical Analysis and prediction System. Monthly Weather Review, v. 123, p. 717-736, 1995.

MEYERS, M. P.; WALKO, R. L.; HARRINGTON, J. Y.; COTTON, W. R.New RAMS cloud microphysics parameterization Part II: the two-moment scheme. Atmospheric Research, v 45, p. 3-39, 1997.

MONCRIEFF, M. W.; KRUEGER, S. K.; GREGORY, D.; REDELSPERGER, J-L.; TAO, W.-K. GEWEX Cloud System Study (GCSS) Working Group 4: precipitating convective cloud systems. Bulletin of the American Meteorological Society, v. 78, n. 5, p. 831-845, 1997.

PIELKE, R. E.; COTTON, W. R.; WALKO, R. L.; TREMBACK, C. J.; LYONS, W. A.; GRASSO, L. D.; NICHOLLS, M. E.; MORAN, M. D.; WESLEY, D. A.; LEE, T. J.; COPELAND, J. H. A Comprehensive Meteorological Modeling System - RAMS. Meteorology and Atmospheric Physics, v.49, p. 69-91, 1992.

RANDALL, D. A.; XU, K-M.; SOMERVILLE, R. J. C.; IACOBELLIS, S. Single-column models and cloud ensemble models as links between observations and climate models. Journal of Climate, v. 9, p. 1683-1697, 1996.

RICKENBACH, T. M. Nocturnal Cloud Systems and Diurnal Variation of Clouds and Rainfall in Southwestern Amazonia. Monthly Weather Review, v. 132, p. 1201-1219. 2004.

SHORT, D. A; KUCERA, P. A.; FERRIER, B. S.; GERLACH, J. C.; RUTLEDGE, S. A.; THIELE, O. W. Shipboard radar rainfall patterns within the TOGA COARE IFA. Bulletin of the American Meteorological Society, v. 78, p. 28172836, 1997.

SMAGORINSKY, J. General circulation experiments with the primitive equations. Part I: the basic experiment. Monthly Weather Review, v. 91, p. 99-164, 1963. 
TAO, W.-K. Cloud resolving Modeling. Journal of the Meteorological Society of Japan, v. 85B, p. 305-330, 2007.

WALKO, R. L.; COTTON, W. R.; MEYERS, M. P.; HARRINGTON, J. Y. New RAMS cloud microphysics parameterization Part I: the single-moment scheme. Atmospheric Research, v. 38, p. 29-62, 1995.

WU, X. Effects of ice microphysics on Tropical radiativeconvective-oceanic-quasi-equilibrium states. Journal of the Atmospheric Science, v. 59, p 1885-1897, 2002.
YOU, Y. Salinity variability and its role in the barrier-layer formation during TOGA COARE. Journal of Phisical Oceonography, v. 25, p. 2778-2807, 1995.

ZHU, H.; HENDON, H.; JAKOB, C. Convection in a parameterized and superparameterized model and its role in the representation of the MJO. Journal of the Atmospheric Science, v. 66, p. 2796-2811, 2009. 